\title{
Saúde bucal e Pacientes com Necessidades Especiais: percepções de graduandos em Odontologia da FOB-USP
}

\author{
Juliana Carvalho Jacomine*; Rafael Ferreira**; Adriana Campos Passanezi Sant'Ana***; Maria \\ Lúcia Rubo de Rezende***; Sebastião Luiz Aguiar Greghi***; Carla Andreotti Damante***; \\ Mariana Schutzer Ragghianti Zangrando***
}

* Estudante de Pós-Graduação, Departamento de Dentística, Endodontia e Materiais Odontológicos, Faculdade de Odontologia de Bauru

** Estudante de Pós-Graduação, Departamento de Prótese e Periodontia, Faculdade de Odontologia de Bauru

*** Docente, Departamento de Prótese e Periodontia, Faculdade de Odontologia de Bauru

Recebido em 09/05/2017. Aprovado em 22/10/2017.

\begin{abstract}
RESUMO
Pacientes com necessidades especiais (PNE) representam uma porcentagem significativa da população brasileira, correspondendo a $24 \%$ da parcela populacional. Entretanto, esses pacientes podem enfrentar diversas barreiras de acesso ao tratamento. O objetivo desse trabalho é conhecer as percepções e dificuldades encontradas por estudantes de Odontologia, referentes ao atendimento de PNE. Foi realizada a aplicação de um questionário aos graduandos do $2^{\circ}$ ao $4^{\circ}$ ano da Faculdade de Odontologia de Bauru $(n=122)$, com questões referentes ao atendimento odontológico e suas percepções sobre os PNE. Observa-se que parcela significativa dos estudantes não se sentem preparados para esse atendimento, chegando a $95 \%$ no $4^{\circ}$ ano. Sobre a percepção quanto ao preparo para atender PNE, os graduandos do $4^{\circ}$ ano referem-se como regular, enquanto a maioria dos que cursam o $2^{\circ}$ e $3^{\circ}$ anos acredita não estar preparada para o atendimento. O tratamento odontológico é fundamental para o estabelecimento da saúde bucal dos pacientes, principalmente quando relacionado aos PNE, sendo essencial o conhecimento das limitações e dos recursos que levam ao acolhimento do paciente por parte dos graduandos. O contato com PNE durante a graduação é extremamente importante para o conhecimento e a ruptura de possíveis preconceitos que possam envolver o atendimento dessa população, a fim de promover saúde bucal e qualidade de vida aos PNE.
\end{abstract}

Descritores: Assistência Odontológica. Saúde Bucal. Síndrome de Down. Estudantes de Odontologia. 
Saúde bucal e Pacientes com Necessidades Especiais: percepções de graduandos em Odontologia da FOB-USP

\section{INTRODUÇÃO}

Pessoas com deficiência podem ser definidas, de acordo com a Convenção sobre os Direitos das Pessoas com Deficiência (2007), como aquelas que têm impedimentos a longo prazo, de natureza física, intelectual (mental), ou sensorial (visão e audição) ${ }^{1}$. Em saúde, este individuo corresponde ao "paciente com necessidade especial" (PNE), sendo todo e qualquer indivíduo que apresente determinados desvios dos padrões de normalidade e que necessite de atenção e abordagem especiais por um período de sua vida ou pela vida toda ${ }^{2}$.

Mais de 45,6 milhões de brasileiros declararam ter alguma deficiência, segundo dados do Censo Demográfico 2010, o que corresponde à aproximadamente $24 \%$ da população brasileira. O maior percentual de pessoas com pelo menos uma das deficiências investigadas (visual, auditiva, mental e motora) reside no Nordeste do país, representando cerca de $26,6 \%$ da população da região ${ }^{3}$.

Em razão de suas inúmeras limitações, sejam elas físicas, mentais e/ou sociais, PNE tendem a apresentar, além do comprometimento sistêmico, agravos bucais mais severos. Dessa forma, a necessidade de uma atenção odontológica especial, com os cuidados específicos de acordo com cada indivíduo tornase extremamente importante ${ }^{4,5}$.

A Síndrome de Down (SD) é um exemplo bastante conhecido que define um tipo de PNE. Constitui uma das causas mais frequentes de deficiência mental, abrangendo também cerca de $18 \%$ do total de deficientes mentais em instituições especializadas ${ }^{6}$. Já o autismo, assunto bastante discutido na atualidade, manifesta-se na primeira infância e é descrito por dificuldades na interação interpessoal e comunicação restrita e até mesmo repetitiva, com alterações comportamentais, funcionais e emocionais ${ }^{7}$.
Entretanto, o panorama da Odontologia atual não reflete a atuação dessa importante especialidade, a Odontologia para Pacientes com Necessidades Especiais (OPNE). Dos 175 cursos de Odontologia do Brasil que participaram de um estudo que avaliava a opinião dos estudantes sobre o atendimento de PNE, 55 retornaram o questionário aplicado e apenas 31 afirmaram oferecer a disciplina de OPNE, seja de forma obrigatória ou optativa ${ }^{7}$. Isso é comprovado quando se considera o limitado número de cursos de Odontologia no Brasil que dispõem de espaço físico e treinamento específico para o tratamento desses pacientes. Assim, o contato com os PNE é mínimo durante a graduação, não tendo os alunos a oportunidade de conhecer e tratar tais pacientes ${ }^{8}$.

Apesar de se ter pleno conhecimento de que PNE apresentam saúde bucal prejudicada ${ }^{9}$, por maior prevalência de algumas doenças bucais $^{10,11}$ devido a fatores imunitários ${ }^{12}$ ou financeiros ${ }^{13,14}$, uma parcela significativa dessa população não possui acesso a tratamentos odontológicos ${ }^{15}$.

Frente ao cenário científico com reduzido número de pesquisas que abordem tais aspectos, o presente estudo avalia a percepção de graduandos em Odontologia do segundo ao quarto ano da Faculdade de Odontologia de Bauru (FOB-USP) quanto às suas experiências e expectativas referentes ao tratamento odontológico de PNE.

\section{METODOLOGIA}

Este trabalho faz parte de um projeto maior aprovado pelo Comitê de Ética em Pesquisa com Seres Humanos da instituição, processo $n^{\circ}$ 116/2010. Foram incluídos no estudo alunos regularmente matriculados no $2^{\circ}$ e no $4^{\circ}$ ano da graduação da FOB-USP.

Utilizou-se um questionário com 9 questões fechadas (figura 1) e foram excluídos do 
Saúde bucal e Pacientes com Necessidades Especiais: percepções de graduandos em Odontologia da FOB-USP

estudo aqueles que se apresentavam incompletos.

Para as questões 6 e 7 foram considerados os seguintes padrões de valores médios referentes ao sentimento frente ao caso clínico: 1 e 2 muito bom; 3 e 4 bom, 5 e 6 regular, 7 e 8 ruim, 9 e 10 péssimo.

Os dados foram tabulados em planilha e analisados por meio de estatísticas descritivas.

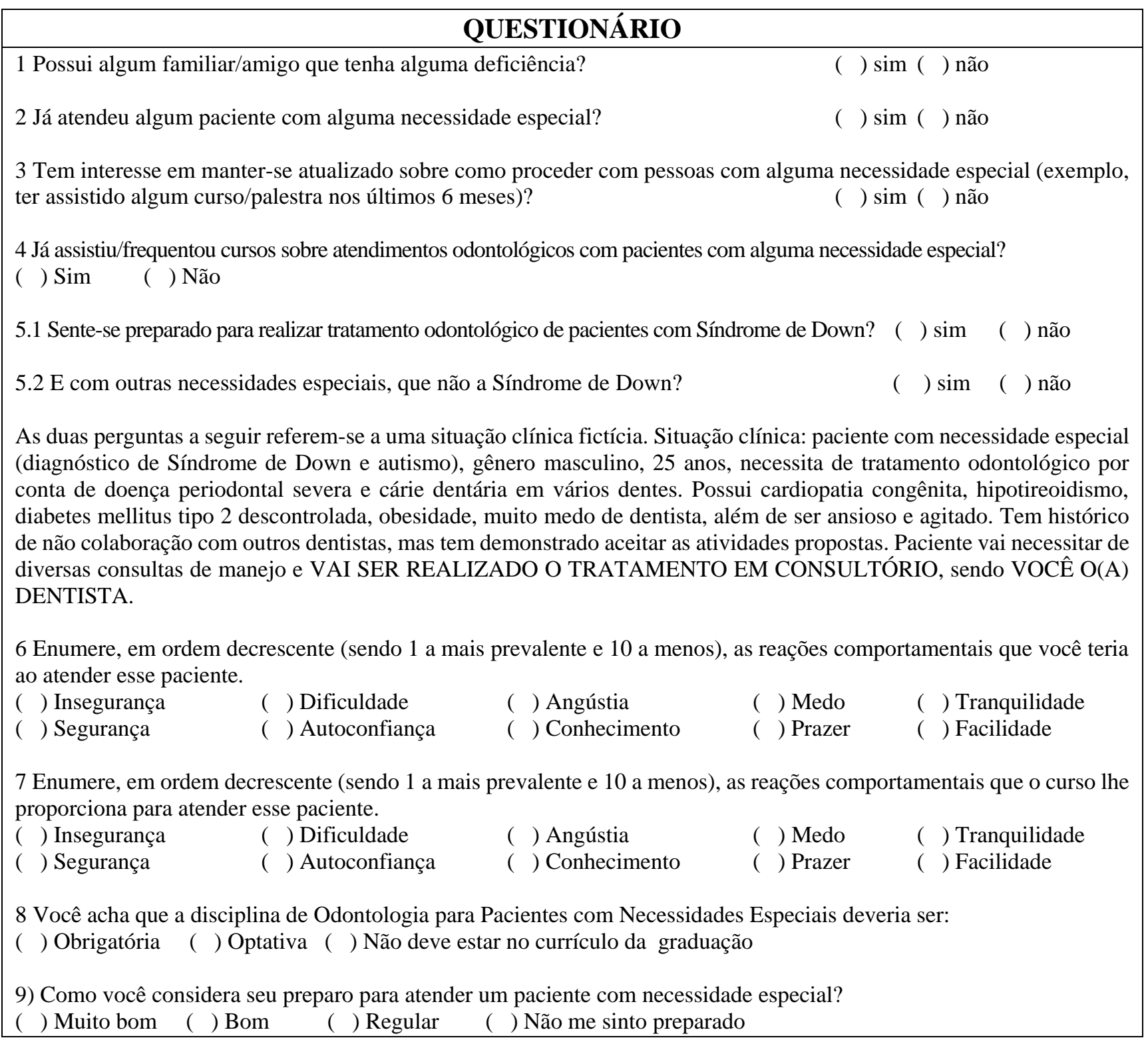

Figura 1. Questionário aplicado aos graduandos de Odontologia

\section{RESULTADOS E DISCUSSÃO}

Dos 132 estudantes incluídos na pesquisa, $122(92,42 \%)$ preencheram totalmente os questionários (47 - 93,61\% do $2^{\circ}$ ano; 45 - 84,44\% do $3^{\circ}$ ano e $40-100 \%$ do $4^{\circ}$ ano). Os estudantes do $4^{\circ}$ ano foram subdivididos em função de terem ou não cursado a disciplina optativa de OPNE na FOB-USP (12 - 30\% e 28 - 70\%, respectivamente). 
A idade dos participantes variou entre $19 \mathrm{e}$ 25 anos, com média de 22 anos. Em relação ao gênero, percebeu-se uma porcentagem significativamente maior de mulheres $(63,93 \%)$.

A maioria dos graduandos não relata convivência com portadores de alguma deficiência, o que é mais marcante entre os alunos do $2^{\circ}$ ano. De forma geral, mais da metade dos estudantes não tem convivência com pessoas com necessidades especiais (tabela 1). Essa ausência de convívio social, se persistente, pode acarretar diversas situações negativas na vida profissional desses estudantes e na disponibilidade de atendimento aos PNE, já que a ausência de informação e contato dos graduandos com essa população durante a faculdade pode influenciar no reduzido atendimento a esses pacientes posteriormente $^{16}$. Assim, a universidade desempenha importante papel de inclusão, bem como mostrar aos alunos uma realidade que lhe é diferente.

De modo geral, observa-se que com o passar dos anos o aluno acaba tendo possibilidade de conhecer/ter contato com essa população dentro da universidade. A porcentagem de alunos que atendeu PNE aumentou conforme o avanço acadêmico do graduando, o que é esperado conforme sua prática clínica vai sendo aperfeiçoada. Os estudantes do $4^{\circ}$ ano foram os que mais atenderam PNE, possivelmente também porque a disciplina de OPNE é ministrada nesse período.

Entretanto, deve-se destacar a abrangência da questão referente à necessidade especial, sendo aqueles pacientes com restrições de capacidades advindas da idade ou agravos adquiridos, resultando em alguma forma de limitação em sua função intelectual, física ou social, e não somente quem possui uma deficiência ${ }^{17}$.

Assim, para futuros estudos, sugere-se a colocação de questões mais específicas, tais como "Já atendeu algum paciente com alteração neurológica (decorrentes de acidente vascular cerebral, dentre outros)?”, “Já atendeu algum paciente com problema psíquico (pacientes com transtorno de humor, dentre outros)?" ou “Já atendeu algum paciente com déficit cognitivo), bem como outras questões que permeiam a área dos PNE, a fim de se compreender o perfil de paciente em questão.

Ao se analisar o interesse dos alunos na atualização em como proceder com pessoas com necessidades especiais, verifica-se que a grande maioria dos estudantes deseja esse conhecimento (tabela 1). Os alunos que estão iniciando a prática clínica ( $2^{\circ}$ ano) demonstram grande interesse $(95,45 \%)$, o que se reduz no ano seguinte $(68,42 \%)$, porém com enorme crescimento no último ano $(97,5 \%)$. O fato de no $3^{\circ}$ ano ter decrescido o percentual de alunos que se interessam por essa atualização pode estar relacionado a viés de confundimento do início da prática clínica, em que os pacientes de Prótese Total (frequentemente idosos com alterações sistêmicas) são também atendidos pelos graduandos. Logo, para eles, não seriam necessárias mais atualizações, visto que são capazes de atendê-los no dia a dia. Isso nos mostra, mais uma vez, a indispensabilidade de especificação da necessidade especial. A vontade de atualização dos alunos é uma possibilidade de experiência/contato clínico com essa parcela da população, o que promove mudanças em diversas fases do futuro profissional. A partir desse contato, o mesmo tem material para observar quais são as dificuldades e vitórias ao se atender esses pacientes. Isso pode ser refletido, por exemplo, na busca de técnicas ou temas ligados a esses pacientes.

Quanto à conclusão da questão anterior, a maioria dos alunos diz já ter frequentado/ assistido cursos sobre o atendimento odontológico de PNE. Entretanto, esses valores acabam sendo menores se relacionados à questão anterior, demonstrando 
que muitos dizem ter interesse, mas parte desses não buscam cursos que os atualizem nesse aspecto (tabela 1). O mesmo pode ser visto quando se analisa essa questão especificamente em relação aos alunos do $4^{\circ}$ ano, em que uma porcentagem menor busca atualização (tabela 1). O baixo número de estudantes que cursaram a disciplina de OPNE se deve ao seu caráter optativo. A disciplina teve sua primeira turma em 2016, com duração de aproximadamente dois meses, disponibilizando apenas 12 vagas e tendo como pré-requisito estar cursando o $4^{\circ}$. ano. Entretanto, a inserção dessa disciplina representa um grande avanço, frente à ampla área de pacientes especiais, estando alunos, comunidade científica e sociedade, beneficiadas com tal ação.

Entretanto, pela menor porcentagem de alunos que realmente busca o aperfeiçoamento no assunto, deve-se ressaltar que essa falta de prática de aperfeiçoamento pode ocorrer por outro motivo: ausência de cursos na área. A disciplina de OPNE é relativamente recente e a oferta de cursos em jornadas e congressos acadêmicos tem sido discreta. Contudo, observa-se o aumento de cursos voltados para a área de Odontologia Hospitalar, que também envolve os PNE, uma vez que por lei deve existir um cirurgião-dentista presente na Unidade de Terapia Intensiva (UTI) ${ }^{18,19}$.

Cursos de manejo, métodos de controle de placa em paciente com necessidade especial, dentre outros assuntos acerca da realidade desses pacientes, devem ser cada vez mais uma realidade em nossa sociedade, uma vez que os PNE não podem ser negligenciados.

Sabendo que a SD é uma das causas mais frequentes de deficiência mental ${ }^{5}$, torna-se importante conhecer a visão do aluno sobre ter ou não confiança para o atendimento de pacientes com essa síndrome. Nesse aspecto, percebe-se que uma maioria bastante significativa dos estudantes, chegando a $95 \%$ dos graduandos do $4^{\circ}$ ano, não se sente preparada para o atendimento de pacientes com SD (tabela 1).

Essa realidade, possivelmente, deve-se ao fato de que os alunos do último ano têm maior contato com pacientes que apresentam complexidade no atendimento odontológico. Assim, consequentemente, possuem maior conhecimento sobre as dificuldades e particularidades no atendimento de um PNE, como um paciente com SD. Além do mais, essa falta de preparo pode estar relacionada com a ausência de atendimento desses pacientes durante a graduação.

Referente a essa mesma questão, um outro aspecto é que a totalidade dos alunos do $4^{\circ}$ ano que cursaram a disciplina optativa de OPNE diz não se sentir preparada para o tratamento odontológico de pacientes com SD (tabela 1). Isso revela que a vivência clínica durante a disciplina de OPNE mostrou-se importante para situar o graduando na realidade dos PNE. Portanto, mesmo um paciente considerado "bonzinho" e de "fácil atendimento", possui diversas dificuldades que podem não ser superadas, dificultando ou inviabilizando o tratamento odontológico.

Já quando se faz a mesma comparação, excluindo a SD, percebe-se que uma parcela um pouco maior de alunos do $3^{\circ}$ e $4^{\circ}$ ano sente-se preparada para esse cuidado, enquanto que no $2^{\circ}$ ano há mais alunos que se sentem despreparados para o cuidado de pacientes com outras necessidades especiais que não a SD (tabela 1).

Além dessa grande representatividade da SD entre os PNE, o autismo é um tema bastante atual e necessário para a discussão. Por isso foram inseridas duas questões (tabela 2) baseadas em um caso clínico descrito no questionário acerca de um paciente com essas duas deficiências (SD e autismo), a fim de se conhecer as reações comportamentais dos estudantes frente ao atendimento do paciente em questão. Ao se analisar essas reações, considerando que a menor média 
seria a reação mais frequente e a maior média a menos frequente, nota-se que entre todos os graduandos, as reações que mais se destacaram foram insegurança e dificuldade (tabela 2). Já no outro extremo, quando se observa as reações menos frequentes, nota-se que facilidade e segurança são o que os alunos menos relatam, também de forma homogênea entre todos os anos (tabela 2).

Quando se avalia, entretanto, as reações comportamentais que o curso proporciona ao aluno ao atender esse mesmo paciente, nota-se que a reação mais prevalente entre os alunos do $2^{\circ}$ e $4^{\circ}$ anos é conhecimento e entre os alunos do $3^{\circ}$ ano a insegurança. Já quanto à reação menos prevalente, percebe-se que facilidade é o que menos representa todos grupos (tabela 2).

Se analisarmos apenas os graduandos do $4^{\circ}$ ano, as reações proporcionadas se aproximam um pouco mais, possuindo médias semelhantes.
Conhecimento é mais prevalente entre os alunos do $4^{\circ}$ ano em geral e entre aqueles que cursaram a disciplina de OPNE. Já para o grupo que não cursou a optativa, a mais prevalente é a dificuldade. Quando se observa a reação menos prevalente para o $4^{\circ}$ ano em geral e para os alunos que não cursaram a optativa, facilidade ganha destaque e, por fim, para os alunos que cursaram a optativa o posto de reação menos prevalente foi a angústia (tabela 2).

Desse modo, ao terminar a graduação na FOB-USP, de forma geral, os alunos revelam possuir conhecimento teórico para o atendimento de PNE, mas ainda assim relatam dificuldade e angústia. Nesse sentido, ações devem ser realizadas para que promovam desenvolvimento integral do aluno, pois o convívio e a prática clínica proporcionam confiança e segurança durante o atendimento odontológico.

Tabela 1. Resposta dos estudantes às questões 1 a 5 , em percentuais

\begin{tabular}{|c|c|c|c|c|c|c|c|c|c|c|}
\hline \multirow[b]{2}{*}{ Questão } & \multicolumn{5}{|c|}{ Sim } & \multicolumn{5}{|c|}{ Não } \\
\hline & $2^{\circ}$ ano & $3^{\circ}$ ano & $\begin{array}{l}4^{\circ} \text { ano } \\
\text { global }\end{array}$ & $\begin{array}{c}4^{\circ} \text { ano com } \\
\text { optativa }\end{array}$ & $\begin{array}{c}4^{\circ} \text { ano sem } \\
\text { optativa }\end{array}$ & $2^{\circ}$ ano & $3^{\circ}$ ano & $\begin{array}{l}4^{\circ} \text { ano } \\
\text { global }\end{array}$ & $\begin{array}{c}4^{\circ} \text { ano com } \\
\text { optativa }\end{array}$ & $\begin{array}{c}4^{\circ} \text { ano sem } \\
\text { optativa }\end{array}$ \\
\hline 1 & 29,5 & 57,9 & 47,5 & 50,0 & 46,4 & 70,5 & 42,1 & 52,5 & 50,0 & 53,6 \\
\hline 2 & 15,9 & 36,8 & 75,0 & 91,7 & 67,8 & 84,1 & 63,2 & 25 & 8,3 & 32,2 \\
\hline 3 & 95,4 & 68,4 & 97,5 & 100,0 & 96,4 & 4,5 & 31,6 & 2,5 & - & 3,6 \\
\hline 4 & 54,5 & 42,1 & 65,0 & 100,0 & 50,0 & 45,5 & 57,9 & 35,0 & - & 50,0 \\
\hline 5.1 & 29,5 & 10,5 & 5,0 & - & 7,1 & 70,5 & 89,5 & 95,0 & 100,0 & 92,9 \\
\hline 5.2 & 22,7 & 18,4 & 20,0 & . & 28,6 & 77,3 & 81,6 & 80,0 & 100,0 & 71,4 \\
\hline
\end{tabular}

Tabela 2. Valores médios das reações comportamentais em relação às questões 6 e 7

\begin{tabular}{|c|c|c|c|c|c|c|c|c|c|c|}
\hline \multirow[b]{2}{*}{ Reação } & \multicolumn{5}{|c|}{$\begin{array}{l}\text { Questão 6. Reações comportamentais que } \\
\text { o estudante teria }\end{array}$} & \multicolumn{5}{|c|}{$\begin{array}{l}\text { Questão 7. Reações comportamentais que } \\
\text { o curso proporciona }\end{array}$} \\
\hline & $2^{\circ}$ ano & $3^{\circ}$ ano & $\begin{array}{l}4^{\circ} \text { ano } \\
\text { global }\end{array}$ & $\begin{array}{l}4^{\mathrm{o}} \text { ano com } \\
\text { optativa }\end{array}$ & $\begin{array}{l}4^{\circ} \text { ano sem } \\
\text { optativa }\end{array}$ & $2^{\circ}$ ano & $3^{\circ}$ ano & $\begin{array}{l}4^{\circ} \text { ano } \\
\text { global }\end{array}$ & $\begin{array}{l}4^{\circ} \text { ano com } \\
\text { optativa }\end{array}$ & $\begin{array}{c}4^{\circ} \text { ano sem } \\
\text { optativa }\end{array}$ \\
\hline Insegurança & 3,52 & 2,76 & 2,42 & 1,66 & 2,75 & 5,44 & 3,76 & 5,27 & 5,58 & 5,10 \\
\hline Dificuldade & 3,54 & 3,44 & 2,92 & 2,75 & 3,00 & 5,25 & 4,23 & 4,70 & 4,91 & 4,60 \\
\hline Angústia & 5,09 & 5,09 & 5,12 & 5,00 & 5,17 & 5,6 & 6,44 & 6,52 & 7,75 & 6,00 \\
\hline Medo & 4,97 & 4,97 & 4,07 & 3,50 & 4,32 & 6,09 & 5,44 & 6,32 & 6,83 & 6,10 \\
\hline Tranquilidade & 5,76 & 5,76 & 6,65 & 7,25 & 6,39 & 5,00 & 5,63 & 4,90 & 5,25 & 4,75 \\
\hline Segurança & 6,79 & 6,79 & 7,12 & 7,25 & 7,07 & 5,06 & 5,78 & 5,20 & 4,58 & 4,46 \\
\hline Autoconfiança & 5,88 & 5,88 & 6,70 & 7,16 & 6,50 & 4,72 & 5,65 & 5,72 & 5,50 & 5,82 \\
\hline Conhecimento & 5,41 & 5,41 & 5,50 & 5,58 & 5,46 & 4,22 & 4,78 & 4,22 & 3,16 & 4,67 \\
\hline Prazer & 4,93 & 4,93 & 5,70 & 5,33 & 5,85 & 4,95 & 5,71 & 5,70 & 4,75 & 6,10 \\
\hline Facilidade & 7,51 & 7,51 & 8,20 & 8,50 & 8,07 & 6,25 & 7,31 & 6,60 & 6,83 & 6,50 \\
\hline
\end{tabular}


Quando se observa a percepção dos graduandos quanto à natureza da disciplina de OPNE, atualmente oferecida como optativa, apenas os alunos do $2^{\circ}$ ano expressam a necessidade de tal disciplina como obrigatória. Metade dos alunos do $3^{\circ}$ e $4^{\circ}$ anos prefere que seja optativa e, curiosamente, no $4^{\circ}$ ano aparece uma única opinião de que a disciplina não deveria estar no currículo (tabela 3). A representatividade desse aluno permite uma interpretação ambígua: ou ele se sente preparado para o atendimento desses pacientes e acredita que o curso já possui disciplinas que supram tal necessidade ou, a partir de uma visão mais negativa, que esses pacientes não farão parte de sua rotina clínica e, logo, não é necessário o aprendizado sobre PNE.

Entretanto, quando se analisam as respostas dessa questão apenas entre os alunos do $4^{\circ}$ ano, observa-se diferenças nas respostas, possivelmente pela experiência e vivência adquiridas durante a disciplina optativa. $\mathrm{O}$ dobro dos alunos que cursou a OPNE percebeu a importância dessa disciplina como obrigatória. A maior parte dos graduandos do $4^{\circ}$ ano que não cursou a disciplina prefere que a mesma continue sendo uma disciplina optativa, por isso a divisão quase pela metade em comparação a todos os graduandos do $4^{\circ}$ ano (tabela 3).

$\mathrm{Na}$ autoavaliação quanto ao preparo do aluno para atender um PNE, nota-se que no $4^{\circ}$ ano a grande maioria diz ter preparo regular, enquanto que a maioria dos alunos do $2^{\circ}$ e $3^{\circ}$ anos diz não estar preparada para esse atendimento (tabela 4). Deve-se destacar, ainda, a importância de se trabalhar desde a graduação com PNE, capacitando o futuro profissional e rompendo inúmeros tabus e conceitos errôneos acerca do assunto. Dessa forma, o tratamento ao paciente com SD seria desmitificado, possibilitando uma abordagem abrangente $\mathrm{e}$ integral, favorecendo a qualidade de vida dos pacientes e a segurança do profissional.

Tabela 3. Respostas dos estudantes (em percentuais) à questão 8 sobre a natureza da disciplina de OPNE

\begin{tabular}{rccc}
\hline Ano & Obrigatória & Optativa & Não deveria estar no currículo \\
\hline $\mathbf{2}^{\mathbf{o}}$ & 56,8 & 43,2 & - \\
$\mathbf{3}^{\mathbf{0}}$ & 50,0 & 50,0 & - \\
$\mathbf{4}^{\mathbf{o}}$ global & 47,5 & 50,0 & $2,5 \%$ \\
$\mathbf{4}^{\mathbf{o}} \mathbf{c o m}$ optativa & 66,7 & $33,3 \%$ & - \\
$\mathbf{4}^{\mathbf{0}} \mathbf{\text { sem optativa }}$ & 39,3 & $57,1 \%$ & $3,6 \%$ \\
\hline
\end{tabular}

Tabela 4. Respostas dos estudantes (em percentuais) à questão 9 sobre a percepção de seu preparo para atender um PNE

\begin{tabular}{r|cccc}
\hline Ano & Muito bom & Bom & Regular & Não se sente preparado \\
\hline $\mathbf{2}^{\mathbf{0}}$ & 0 & $22,7 \%$ & $36,4 \%$ & $40,9 \%$ \\
$\mathbf{3}^{\mathbf{0}}$ & 0 & $7,9 \%$ & $36,8 \%$ & $55,3 \%$ \\
$\mathbf{4}^{\mathbf{o}}$ global & 0 & $10 \%$ & $72,5 \%$ & $17,5 \%$ \\
$\mathbf{4}^{\mathbf{0}} \mathbf{~ c o m ~ o p t a t i v a ~}$ & 0 & $16,5 \%$ & $75 \%$ & $8,5 \%$ \\
$\mathbf{4}^{\mathbf{0}}$ sem optativa & 0 & $7,2 \%$ & $67,8 \%$ & $25 \%$ \\
\hline
\end{tabular}


A preparação de graduandos para atuar com PNE pressupõe, além de formação técnica, a sensibilização e a predisposição ao atendimento. Tendo-se em vista a situação atual de implantação de políticas inclusivas, é provável que alunos de graduação em odontologia tenham tido experiências variadas e, possivelmente, contraditórias em relação a contatos com PNE em situações de inclusão. Para melhor planejar as aulas e atividades clínicas relativas à disciplina de OPNE, é importante conhecer formas de pensar e sentir, bem como as tendências referentes à ação dos estudantes de Odontologia, no que tange o atendimento inclusivo de pacientes com deficiência ${ }^{20}$.

Com os resultados, observa-se que o preparo dos graduandos da FOB-USP pode ser definido como "regular". Portanto, mesmo com a disciplina optativa de OPNE da instituição, mais ações devem ser realizadas a fim de permitir uma melhor vivência e experiência para os alunos. Neste sentido, tornar obrigatória a disciplina de OPNE, com maior abrangência das deficiências (abordando os diversos tipos de pacientes, como os com alterações neurológicas, psíquicas, cardiopatas, coagulopatas, dentre outros), pode ser uma ferramenta que auxiliaria na formação integral e humanista do cirurgião-dentista egresso desta instituição.

É imprescindível mudar essa realidade, visto que o perfil do profissional definido nas atuais Diretrizes Curriculares Nacionais para os cursos de Odontologia é de um indivíduo com formação generalista, técnica, científica e humanista, com capacidade crítica e reflexiva, preparado para atuar, pautado na ética, no processo de saúde-doença e nos diferentes níveis de atenção, inclusive com PNE. Deve-se objetivar, assim, a integralidade da assistência, com senso de responsabilidade social e compromisso com a cidadania ${ }^{21}$.

\section{CONCLUSÃO}

Os graduandos da FOB-USP não se sentem integralmente aptos a atenderem PNE. Dessa forma, mais ações devem ser realizadas a fim de permitir melhor vivência e experiência, incluindo os PNE na prática clínica, visando à formação de profissionais generalistas, capacitados ao atendimento nos diferentes níveis de atenção à saúde, a fim de promover qualidade de vida a esses pacientes.

\section{AGRADECIMENTOS}

Agradecemos à FAPESP (Fundação de Amparo à Pesquisa do Estado de São Paulo) pela concessão da Bolsa de Mestrado (processo 2013/05382-9), bem como do Auxílio à Pesquisa (Processo 2013/16607-1) ao bolsista Rafael Ferreira e a Profa. Dra. Carla Andreotti Damante, respectivamente, no projeto de pesquisa que deu origem a esse trabalho.

\section{ABSTRACT \\ Oral health and patients with special needs: perception of undergraduates in Dentistry of FOB-USP}

Patients with special needs (PSN) represent a significant part of the Brazilian population, corresponding to $24 \%$. However, these patients may face several barriers to access dental care. This study aimed to evaluate the perception of Dental students about PSN treatment. A questionnaire was applied to undergraduates from the 2nd to 4th year of the Faculty of Dentistry of Bauru $(n=122)$, with questions regarding dental care and their perceptions about PSN. It is observed that a significant number of students do not feel prepared for this service, reaching $95 \%$ in the 4th year. Regarding the perception regarding the preparation to treat PSN, the 4th year students are referred to as regular, while the majority of students in the 2 nd and 3rd years believe that they are not prepared. Dental treatment is fundamental for the establishment of oral health of patients, mainly when related to PNE, being essential knowledge of limitations and resources that lead to the reception of the patient by graduates. The contact with PNE during the graduation is essential for the knowledge and the rupture of possible prejudices that can 
involve the attendance of this population, in order to promote oral health and quality of life to the PSN.

Descriptors: Dental Care. Down Syndrome. Oral Health. Dental Students.

\section{REFERÊNCIAS}

1. Convenção sobre os Direitos das Pessoas com Deficiência (2007). Convenção sobre os Direitos das Pessoas com Deficiência: Protocolo Facultativo à Convenção sobre os Direitos das Pessoas com Deficiência: decreto legislativo $\mathrm{n}^{\circ} 186$, de 09 de julho de 2008: decreto $n^{\circ} 6.949$, de 25 de agosto de 2009. - 4. ed., rev. e atual. - Brasília: Secretaria de Direitos Humanos, Secretaria Nacional de Promoção dos Direitos da Pessoa com Deficiência, 2011.

2. Mugayar L. Pacientes especiais. In: klatchoian DA. Psicologia Odontopediátrica. 2. ed. São Paulo: Santos; 2002. p. 197-241.

3. Censo 2010 - Pessoas com Deficiência / Luiza Maria Borges Oliveira / Secretaria de Direitos Humanos da Presidência da República (SDH/PR) / Secretaria Nacional de Promoção dos Direitos da Pessoa com Deficiência (SNPD) / Coordenação-Geral do Sistema de Informações sobre a Pessoa com Deficiência; Brasília: SDH-PR/SNPD, 2012.

4. Dall'magro AK, Dall'magro E, Kuhn GF. Perfil clínico dos pacientes especiais tratados sob anestesia geral no Hospital São Vicente de Paulo de Passo Fundo entre os anos de 2005 e 2010. RFO UPF. 2010;15(3):253-6.

5. Fukuoka CY, Michel-Crossato E, Martins Filho IE, Biazevic MGH, Zaitter WM. Accessibility to dental care for patients with special needs. RSBO. 2011; 8(3):277-81.

6. Nettina SM. Manual de prática de enfermagem. 6. ed. Rio de Janeiro: Guanabara Koogan; 1999.

7. Karst JS, Van Hecke AV. Parent and family impact of autism spectrum disorders: a review and proposed model for intervention evaluation. Clin Child Fam Psychol Rev. 2012;15(3): 247-77.

8. Fassina AP. Análise das disciplinas de pacientes portadores de necessidades especiais nas Faculdades no Brasil em 2005. São Paulo. Dissertação [Mestrado em Odontologia] - Faculdade de Odontologia, Universidade de São Paulo; 2006.

9. Cruz S, Chi DL, Huebner CE. Oral health services within community-based organizations for young children with special health care needs. Spec Care Dentist. 2016; 36(5): 243-53.

10. Newacheck PW, McManus M, Fox HB, Hung YY, Halfon N. Access to health care for children with special health care needs. Pediatrics. 2000; 105(4):760-66.

11. Chi DL, Rossitch KC, Beeles EM. Developmental delays and dental caries in low-income preschoolers in the USA: a pilot cross- sectional study and preliminar explanatory model. BMC Oral Health. 2013; 12(13):53.

12. Thikkurissy S, Lal S. Oral health burden in children with systemic diseases. Dent Clin North Am. 2009; 53(2): 351-7.

13. Chen AY, Newacheck PW. Insurance coverage and financial burden for families of children with special health care needs. Ambul Pediatr. 2006; 6(4):204-9.

14. Schultz ST, Shenkin JD, Horowitz AM. Parental perceptions of unmet dental need and cost barriers to care for developmentally disabled children. Pediatr Dent. 2001; 23(4):321-5.

15. Williams JJ, Spangler CC, Yusaf NK. Barriers to dental care access for patients with special needs in an affluent metropolitan community. Spec Care Dentist. 2015; 35(4):190-6.

16. Rapalo DM, Davis JL, Burtner P, Bouldin ED. 
Cost as a barrier to dental care among people with disabilities: a report from the Florida behavioral risk factor surveillance system. Spec Care Dentist. 2010;30(4):133-9.

17. Santos MTBR, Haddad AS. Quem são os pacientes com necessidades especiais? In: Cardoso RJA, Machado MEL. Odontologia: arte e conhecimento. São Paulo: Artes médicas; 2003. p. 263-8.

18. Brasil. Agência Nacional de Vigilância Sanitária. Resolução-RDC nº, de 24 de fevereiro de 2010. Dispõe sobre os requisitos mínimos para funcionamento de Unidades de Terapia Intensiva e dá outras providências. Diário Oficial da União, 25 fev 2010; Seção 1.

19. Brasil. Senado Federal - Projeto de Lei da Câmara $n^{\circ} 34$, de 2013. Torna obrigatória a prestação de assistência odontológica a pacientes em regime de internação hospitalar, aos portadores de doenças crônicas e, ainda, aos atendidos em regime domiciliar na modalidade home care. Senado Federal 2013. Em tramitação. [Acesso em 4 nov 2016]. Disponível em: http://www25.senado.leg.br/ web/atividade/materias/-/materia/112975.
20. Moraes ABA, Batista CG, Lombardo I, Horino LE, Rolim GS. Verbalizações de alunos de odontologia sobre a inclusão social de pessoas com deficiência. Psicol Estud. 2006; 11(3):607-15.

21. Brasil. Conselho Nacional de Educação. Câmara de Educação Superior. Resolução CNE/CES 3, de 19 de fevereiro de 2002. Institui Diretrizes Curriculares Nacionais do Curso de Graduação em Odontologia. Diário Oficial da União 4 de mar 2002; Seção 1.

\section{Correspondência para:}

Mariana Schutzer Ragghianti Zangrando

e-mail: mariana@ fob.usp.br

Departamento de Prótese, Disciplina de Periodontia
Al. Octávio Pinheiro Brisolla, 9-75
17012-901 Bauru/SP 